\title{
872 PD1 X TGFBR2 AND CD5 X TGFBR2 BISPECIFICS SELECTIVELY BLOCK TGFBR2 ON TARGET-POSITIVE T CELLS, PROMOTE T CELL ACTIVATION, AND ELICIT AN ANTI-TUMOR RESPONSE IN SOLID TUMORS
}

Gregory Moore*, Suzanne Schubbert, Christine Bonzon, Kendra Avery, Rumana Rashid, Erik Pong, Lukasz Ochyl, Alex Nisthal, Seung Chu, James Ernst, John Desjarlais. Xencor, Inc., Monrovia, CA, USA

Background TGFbeta production by solid tumors and their microenvironment is a major mechanism used by tumors to avoid immunosurveillance. Blockade of TGFbeta has been shown to promote an anti-tumor response; however, systemic blockade of TGFbeta has also been associated with toxicity. We hypothesized that a T cell-targeted TGFbR2 bispecific antibody could selectively block the suppressive activity of TGFbeta on $\mathrm{T}$ cells and enhance their anti-tumor activity while avoiding toxicity associated with systemic blockade.

Methods We engineered bispecific antibodies that simultaneously engage PD1 (activated) or CD5 (pan T) and block TGFbR2 using Xencor's XmAb ${ }^{\circledR}$ platform. The anti-TGFbR2 arm was tuned for optimal activity by introducing affinitymodulating amino acid substitutions. The activity of TGFbR2 bispecifics was evaluated in vitro using a signaling assay to measure phosphorylated SMAD (pSMAD) by flow cytometry with exogenous TGFbeta in unactivated and activated PBMC. In vivo activity was evaluated by monitoring the engraftment of human PBMC in NSG mice (huPBMC-NSG). Anti-tumor activity was assessed in huPBMC-NSG mice engrafted with established human cancer cell lines.

Results TGFbR2 bispecifics were confirmed to bind PD1 or CD5 and block binding of TGFbeta to TGFbR2. In vitro, we found that $\mathrm{T}$ cells from serum-deprived PBMC exhibited robust induction of pSMAD in response to TGFbeta, and TGFbR2 bispecifics selectively inhibited pSMAD induction in target-positive $\mathrm{T}$ cells as demonstrated by over a 100-fold potency increase compared to an untargeted anti-TGFbR2 control. Additionally, we saw an enhancement of potency when evaluating activity in target-high $\mathrm{T}$ cells versus target-low or negative immune cells. Intriguingly, CD5-targeted TGFbR2 bispecifics allowed for the targeting of a broader population of T cells compared to PD1-targeting while still conferring potent selectivity against target-negative cells. In vivo, treatment of huPBMC-NSG mice with TGFbR2 bispecifics promoted superior $\mathrm{T}$ cell engraftment. Furthermore, TGFbR2 bispecific treatment of huPBMC-NSG mice containing established MDA-MB231 triple-negative breast cancer tumors promoted an antitumor response that was augmented with PD1 blockade.

Conclusions PD1 $\mathrm{x}$ TGFbR2 and CD5 x TGFbR2 bispecific antibodies were engineered to selectively block TGFbR2 on target-positive $\mathrm{T}$ cells and evaluated in vitro and in vivo. These observations are compelling and suggest that development of these bispecifics is warranted for the treatment of human malignancies.

http://dx.doi.org/10.1136/jitc-2021-SITC2021.872 